\title{
Dedication to Degradation: The Beauty of Materials Designed to Lay in Ruin
}

\author{
JOHN A. NYCHKA ${ }^{1,3}$ and JAIME KRUZIC ${ }^{2}$ \\ 1.-Department of Chemical and Materials Engineering, University of Alberta, Edmonton, \\ AB T6G 2V4, Canada. 2.-Department of Mechanical Engineering, Oregon State University, \\ Corvallis, OR 97331, USA. 3.-e-mail: jnychka@ualberta.ca
}

\begin{abstract}
Degradation of materials is typically perceived to be a negative response in service. Many designs, and materials, have been and are ruined due to corrosion, fatigue, weathering, ultraviolet light, fungal attack, bacterial attack, erosion, wear, electromigration... and on the list goes. However, the carefully controlled and purposeful degradation of materials is a prerequisite for success for some designs-and such ability is a beautiful necessity when it comes to many regenerative biomaterials. In other instances, we must seek first to understand the degradation mechanisms before we can achieve degradation prevention-and the resistance of some materials to degradation is also beautiful. Regardless of whether we try to prevent or elicit degradation, our dedication to degradation of materials is ever present in materials design.
\end{abstract}

No matter what things you study, you will always find that those which are good and useful are also graced with beauty.

Baldassare Castiglione, Cortegiano, 1528.

Some designs "wear in" and some designs "wear out," and in many cases, the favored outcome is dictated by the material(s) of choice. For example, automobile tires wear away over time, which is undesirable, and their performance has been optimized through careful materials engineering. A new pair of leather shoes will soften to mold to one's foot and become more comfortable, which is desirable. In both cases, the material responds to its service conditions and changes: the tire rubber heats up due to friction with the driving surface, and the rubber becomes less stiff and more prone to wear; the shoes are bent repeatedly, and the moisture from perspiration also acts to hydrate the collagen within the leather to allow it to rearrange and become more compliant. Material choice affects design. This review discusses some concepts that underpin materials design with respect to our dedication to degradation. The four articles in this

John A. Nychka and Jaime Kruzic are the guest editors for the Biomaterials Committee of both the TMS Electronic, Magnetic, \& Photonic Materials and the TMS Structural Materials divisions, and coordinators of the topic Degradation of Biomaterials in this issue. technical topic elaborate on the concept of materials design with degradation at the forefront; designing materials to behave differently is critical in our development of new healthcare advances or in the protection of biological resources.

Natural materials degrade, and they have been designed that way. Through the degradation of natural materials, there is a replenishment of the earth through return of raw materials. Beings using the earth, or its derivatives, as a food source recycle previously used elements to create more life. Nature is the ultimate consumer-there is very little waste from one organism that is not used by another. Such is sustainable design, and it can only be accomplished by the degradation of materials to maintain a relatively constant stock of elements.

The very materials of life are transient; we borrow our atoms for a finite period of time. Degradation is thus a recovery process, a lifecycle (cradle to cradle) relying on thermodynamic, electrochemical, and biological processes. Degradation can permit many subsequent processes to occur that are especially beneficial in nature, and in our technical world, biomaterials rely on degradation as well. For 
example, in vitro or in vivo degradation of bone tissue scaffolds mimics how bone is resorbed by cells (osteoclasts). And such "bone resorption" cells make it possible for the complete regeneration of the tissue via "bone forming" cells (osteoblasts) every 7-10 years or so in the body. So why should our bones remodel? Well, simply put, our bodies must repair microdamage caused by normal wear and tear, fix fractures, and adjust the structure of the tissue to minimize stresses from load-bearing physical demands. Bone is a naturally smart selfrepairing material, but it remodels at a slow pace. As scientists and engineers we are tasked with speeding up the process without any other impact, and such a challenge is complicated.

Technical materials, such as bioactive ceramic glasses, have been used for bone remodeling in vivo with great success, and interestingly they completely resorb (i.e., exhibit controlled degradation). However, although the bone remodeling properties of bioactive glasses are very desirable, their mechanical properties are poor-fracture toughness is low. When one imagines implanting a material to replace or regenerate bone, a constraint would be that the material is being used to replace a structural element in the skeletal system and a brittle material would not be a wise choice; thus, bioactive glasses are limited in their use as powders for bone defect filling and in some cases for small bones in the ear. Figure 1 displays the microstructure of a partially devitrified bioceramic glass (bioactive glass 45S5). The spherical regions are crystals that have been intentionally grown in the amorphous matrix during heat treating to produce crack deflectors within the glass. While some gains in fracture toughness can be achieved through such materials processing, the bioactive response is not drastically altered. Hence, balancing controlled

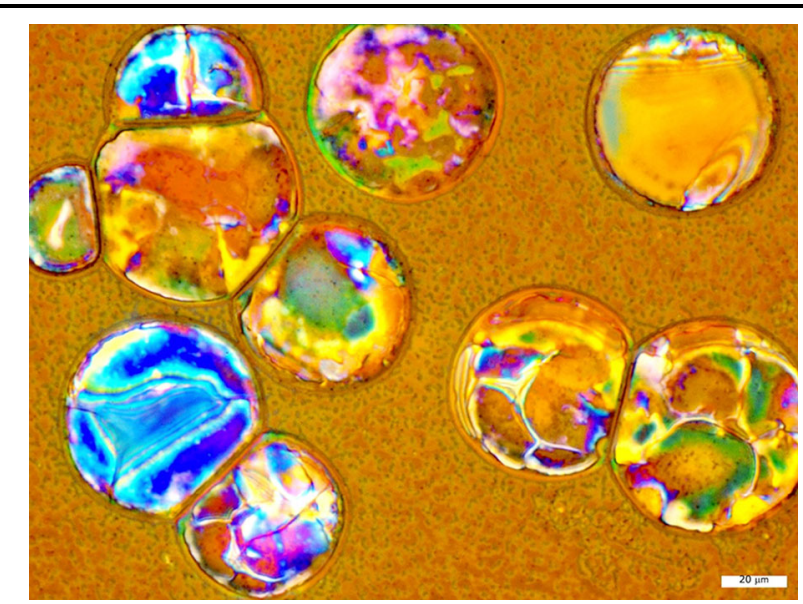

Fig. 1. Spherical crystallites of combeite growing in a bulk amorphous matrix of bioactive glass $45 S 5$ (polarized light bright field micrograph). The spherical particles aid the fracture resistance of the material via crack deflection and yet do not drastically inhibit in vitro surface mineralization. Image: S. Ksashyap and J.A. Nychka. degradation with other performance criteria such as mechanical response is complex.

Controlled degradation is actually not an easy characteristic to master. If you have ever seen bones left out in the sun, you will have noted that they will last for a very long time-they do not degrade readily. However, those same bones if still alive would be self-degrading constantly. In this issue, Winkler et al. discuss how bone remodeling occurs and present results measuring how the bone cells responsible for bone destruction (osteoclasts) degrade a bone tissue scaffold using microcomputed tomography. The article conveys the biological nature of constructive and destructive tendencies of bone tissue based on the cell type. A common misconception is that the body is templated to grow and not degrade itself, but Winkler et al.'s article ("Three-Dimensional Quantification of Calcium Salt-Composite Resorption (CSC) in vitro by MicroComputed Tomography (Cicro-CT)" by T. Winkler, X.Y. Dai, G. Mielke, S. Vogt, H. Beuchner, J.T. Schants, Y. Harder, H.G. Machens, M.M. Morlock, and A.F. Schilling) demonstrates (I) the importance of degradation and (II) that the manner in which bone is remodeled can be measured in vitro rather than in vivo. These outcomes create the potential for using fewer animal models in the prescreening of bone tissue scaffolds while gaining a better understanding of how osteoclasts will affect materials once they are placed in vivo.

In the article titled "High-Strength-Low-Alloyed (HSLA) Mg-Zn-Ca Alloys with Excellent Biodegradation Performance" by J. Hofstetter, M. Becker, E. Martinelli, A.M. Weinberg, B. Mingler, H. Kilian, S. Pogatscher, P.J. Uggowitzer, and J.F. Löffler, the authors attack the difficult problem of balancing the rate of resorption of magnesium-based bone tissue implants with mechanical properties. This "goldilocks"-style problem is a fine balance between the desire to get the degradation "just right" so that the implant can perform its function to regenerate bone tissue and avoid hydrogen-induced damage in remodeled tissues caused by corrosion reaction products. The MgZnCa alloys described can exhibit rather drastic differences in degradation rate based on small changes in chemistry.

Pichler et al. also consider the body's response to degrading materials within the body through their study on the immunological response to biodegradable magnesium implants ("Immunological Response to Biodegradable Magnesium Implants" by Karin Pichler, Stefan Fischerauer, Peter Ferlic, Elisabeth Martinelli, Hans-Peter Brezinsek, Peter J. Uggowitzer, Jörg F. Löffler, and Annelie-Martina Weinberg). Again we see that alloy chemistry plays a crucial role in performance and that Mg-based implants can actually improve the innate immune system under certain conditions.

Finally, in a different application discussed in the article "Degradation of Lignocellulosic Materials and Its Prevention" by Jeffrey Morrell, the author 


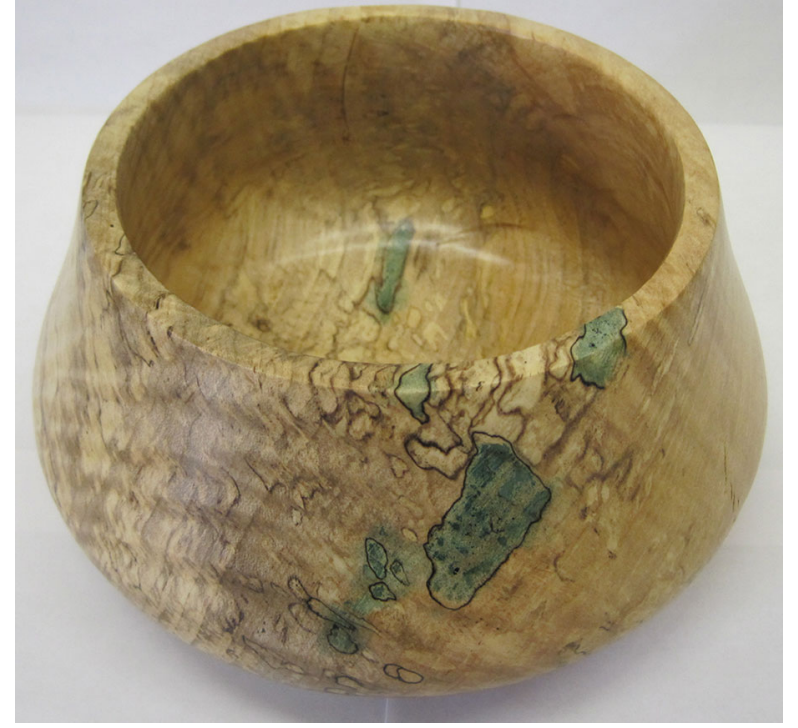

Fig. 2. A bowl made from curly soft maple with beautiful features of white rot, zone lines, and green stain produced by spalting. Image: Sara C. Robinson, Oregon State University.

discusses an attack of lignocellulosics (wood-based materials) with intent to avoid degradation through various treatments. Wood can take many years to degrade depending on the location, species of wood, and amount of water. Interestingly, different species of wood degrade at different rates, and one cannot help but wonder why. Could it be that the locations have different rainfall, soil conditions, temperature fluctuations, relative humidity, and pests, or could it be that the ecosystem has found a balance in which the species' lifetimes have adjusted to reach a compromise? As Charles Darwin recounted, nature is in a "struggle for existence" and the fact that the earth is not consumed by the progeny of one specie is because predation and degradation occur. Humans are a "predator" or user of wood: more than half the wood harvested globally is used for fuel-in which the energy stored in the wood can only be released through combustion, which is basically a degradation mechanism of wood to produce heat in our struggle for existence. Finally, despite the importance of avoiding wood degradation in our everyday environment, it is interesting to note that wood degradation by specific fungi is often used for staining and decorating wood objects and art by a process called spalting, as is shown for the bowl in Fig. 2.

Designing materials to degrade at first glance may appear to relegate them to lay in ruin. However, when such a "ruin" is controlled to make new human bone tissues and repairs, improve our innate immune system, or decorate the objects in our world, then such ruins are likely to be welcomed and appreciated for their beauty.

\section{REFERENCES}

1. C. Darwin, On the Origin of Species (London: W. Clowes and Sons, 1859). 